\title{
Video-STM, LEED and X-ray diffraction investigations of PTCDA on graphite
}

\author{
C. Ludwig ${ }^{1}$, B. Gompf ${ }^{1}$, W. Glatz ${ }^{1}$, J. Petersen ${ }^{1}$, W. Eisenmenger ${ }^{1}$, M. Möbus ${ }^{2}$, U. Zimmermann ${ }^{2}$, and N. Karl ${ }^{2}$ \\ ${ }^{1}$ 1. Physikalisches Institut, Universität Stuttgart, W-7000 Stuttgart 80, Federal Republic of Germany \\ ${ }^{2}$ 3. Physikalisches Institut, Universität Stuttgart, W-7000 Stuttgart 80, Federal Republic of Germany \\ Received August 16, 1991; revised version October 23, 1991
}

Thin films of the organic molecule perylene-3,4,9,10tetracarboxylic-dianhydride ("PTCDA") on graphite (0001) have been investigated from the mono- to the multilayer regime with low energy electron diffraction (LEED), X-ray-diffraction in Bragg-Brentano geometry, and high resolution scanning tunneling microscopy (STM). These different methods proved epitaxial growth in a coincident superstructure and yielded congruent results concerning details of the crystallographic structure of the epilayer. In addition it was possible to resolve submolecular structures in high resolution STM images; a comparison of the 10 resolved maxima of the tunneling current with the molecular structure leads us to question the conventional model description of tunneling.

\section{Introduction}

One route to incorporate organic molecular materials as active electronic, opto-electronic and electro-optic components in modern integrated logic devices is their use as crystallographically well ordered and eventually laterally micro-structured thin films, possibly on semiconductor surfaces. Organic molecules which are potentially useful for this purpose are required to combine interesting properties, like photoconductivity or optical nonlinearity, with thermodynamic and photochemical stability as well as with a large lattice binding energy, i.e. with a sufficiently large molecular weight and/or polarity to provide a sufficiently low vapor pressure.

The aim of the present work has been to study and understand the microscopic conditions under which epitaxial molecular thin films, ranging from the monolayer to the multilayer regime, can be reproducibly prepared and analyzed. Important parameters to optimize or control are steric aspects, such as molecular size and shape, intermolecular interactions, the molecule - substrate interaction, lattice constant match, and kinetic as well as equilibrium thermodynamic system parameters. Literature reports on these topics have been scarce so far.
Adsorbate - substrate interactions of the large organic - molecule perylene-3,4,9,10-tetracarboxylic-dianhydride ("PTCDA") with $\operatorname{Si}(111) 7 \times 7$ have been studied in the references [1] and [2] by NEXAFS and ARUPS investigations. In addition, structural aspects of adsorbatesubstrate interactions have been reported in a number of low energy electron diffraction (LEED) and scanning tunneling microscopy (STM) studies of other aromatic molecules, such as benzene [3,4] and naphthalene [5-7], perylene and coronene [8], and of several phthalocyanines $[9,10]$ on various metal surfaces with different reactivities and symmetries. Examples showing the dependence of molecular order on the size of the molecules are LEED-investigations of $n$-paraffines of different length on a $\mathrm{Pt}(111)$-surface $[11,12]$ and of coronene and hexa-peri-benzocoronene on graphite $(0001)$ and $\mathrm{MoS}_{2}(0001)$ [13]. These experiments also proved that in LEED experiments beam damage, though present, does not in principle prohibit investigations of organic monolayers, if a suitable registration technique is used. For molecular imaging by STM the adsorbates must be sufficiently immobile on short time scales, either owing to packing constraints (close packing), or to strong enough binding interactions between the molecule and the substrate, while longer time scale molecular dynamics can be detected by STM [14].

In this paper we study the structure and epitaxy of thin films of the organic aromatic model compound PTCDA (for the molecular structure see Fig. 5), vapor deposited onto a graphite (0001) surface, by video-scanning tunneling microscopy, low energy electron diffraction, and $\mathrm{X}$-ray diffraction investigations.

PTCDA has been chosen for several reasons: It is, first of all, a fairly large organic molecule with vanishing room temperature vapor pressure, which is commercially available and used as a photochemically stable dye pigment. The material displays sufficient thermal stability to allow purification by sublimation techniques at the necessary high temperature $\left(\sim 400^{\circ} \mathrm{C}\right)$ and vapor deposition by molecular beam epitaxy. Crystalline PTCDA is a photoconductor with potential applications in molecular 
electronics [15-17]. Its crystal structure, both of the monoclinic $\alpha-[18]$ and of the monoclinic $\beta$-modification [19], is unique in that the two symmetry-equivalent molecules lie flat on the (102) plane and form stacks with strong $\pi$-electron molecular interactions in the third dimension. When only the positions of the molecular centers are considered, a quasi-hexagonal symmetry results which was expected to favour epitaxial growth on the (0001) surface of hexagonal graphite. The dipole moments of the strongly electronegative carboxylic anhydride side groups cancel for reasons of molecular symmetry, but the molecule carries a substantial quadrupole moment. Moreover, PTCDA is an electron acceptor-type molecule, a fact which is also reflected by a comparatively large solid state threshold energy for photoelectron emission. $I_{c}^{\text {th }}=6.15(6) \mathrm{eV}$ [27], while graphite is donor-like, $I_{c}^{\text {th }}=4.6 \mathrm{eV}$, thus giving rise to charge transfer interactions. It could be expected, therefore, that flat lying molecules of this kind of molecular architecture experience an interaction with a graphite substrate surface which is substantially increased over that of the respective unsubstituted, nonpolar parent aromatic hydrocarbon (perylene in the case considered, $I_{c}^{\text {th }}=5.12(7) \mathrm{eV}$ [27]). Finally, the question, how details of observed tunneling images relate to the molecular structure, was an additional aspect which prompted the work to be presented here.

\section{Experimental}

We have designed and operated a scanning tunneling microscope which is extremely insensitive to vibrations, has good thermal stability and procudes images in the constant height mode of the scanning tip at nearly video frame rate ( 20 frames $/ \mathrm{s}$ ). The main advantage of the high scanning speed is, that the signal information is transferred to higher frequencies where the $1 / f$-noise of the tunneling current is negligible. In combination with a fast signal averager the instrument is able to produce high resolution images even at extremely noisy conditions. The STM investigations were carried out in air at room temperature with Pt-Ir tips; all STM images shown in this paper are the unfiltered raw data without any further manipulation, except for on-line averaging of a number of subsequent frames. The STM images were photographed directly from the (curved) monitor screen and are therefore slightly disturbed by aberrations.

The LEED experiments were carried out in an ultrahigh vacuum chamber [13] (base pressure $<1 * 10^{-9} \mathrm{mbar}$ ) equipped with electron optics for low energy electron diffraction (OMICRON Comp.). The LEED patterns were photographed with a conventional photocamera from the screen of an image intensifier placed between the LEED window and the camera. The LEED electron beam current thus could be kept smaller than $0.05 \mu \mathrm{A}$. Under these conditions the LEED patterns were stable for $\sim 1 \mathrm{~h}$.

For X-ray diffraction studies in Bragg-Brentano geometry and for fixed $2 \Theta$ /variable $\Theta$ rocking curve analysis a Siemens D 500 diffractometer was used with
$\mathrm{Cu}-\mathrm{K}_{\alpha}$ radiation obtained from a conventional $\mathrm{X}$-ray tube.

PTCDA was purified by a temperature-gradient sublimation technique. The purity was checked by mass-spectrometry and judged as $299 \%$. For molecular beam epitaxy the organic material was evaporated from a copper or graphite crucible at a pressure between $1 \cdot 10^{-8}$ and $3 \cdot 10^{-8}$ mbar onto a freshly cleaved graphite substrate heated to a temperature between 70 and $130^{\circ} \mathrm{C}$. Typical evaporation rates were $\sim 2 \AA /$ min at an evaporation temperature of about $420^{\circ} \mathrm{C}$. Before starting the vapor deposition, the crucibles containing the organic material were outgassed for a short time at a temperature slightly higher than the final evaporation temperature, while the substrate was screened by a shutter.

Natural graphite single crystals were used for the LEED experiments, highly oriented pyrolytic graphite (HOPG) for the STM investigations. These samples were cleaved in air and immediately brought into vacuum. Before film preparation the graphite substrate was heated to $\sim 500^{\circ} \mathrm{C}$ and the single crystallinity checked by LEED.

\section{Results}

\subsection{Scanning tunneling microscope images}

In the slow scan, constant current mode it is possible to image a large area of the substrate. Figure 1 shows a characteristic $500 \AA \times 500 \AA$ image of PTCDA on graphite with two domains of the regular superstructure. The darker right part of the image represents unresolved clean graphite. The maximum height is about $3 \AA$, which leads to the conclusion that the image is that of an epitaxially grown monomolecular layer of flat lying PTCDA mole-

Fig. 1. STM image of an epitaxially grown monolayer of PTCDA on graphite (0001) with two domains (size of the imaged area: $500 \AA \times 500 \AA ; U=0.18 \mathrm{~V}, I=0.5 \mathrm{nA}$, constant current mode) 


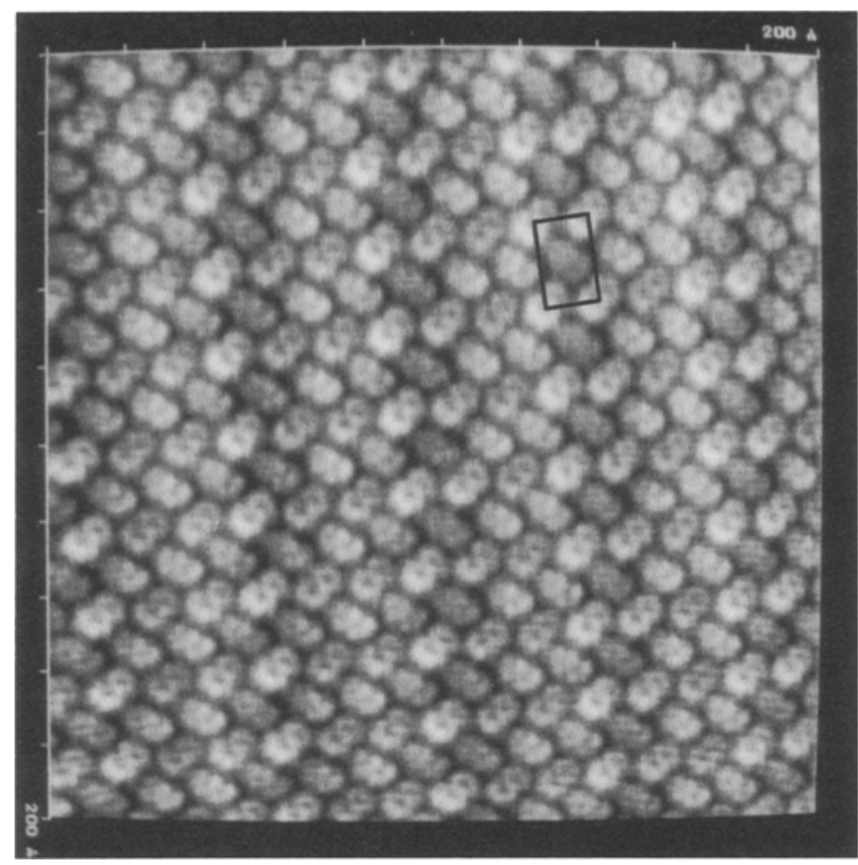

Fig. 2. High resolution Video-STM image of a PTCDA monolayer on graphite (0001). The elongated shape of the individual PTCDA molecules can clearly be recognized. The rectangle marks the unit cell of the periodic structure. In the $a^{\prime}$ direction every third molecule is brighter; $(200 \AA \times 200 \AA, U=0.3 \mathrm{~V}, I=1 \mathrm{nA}$; the image, taken in constant height mode, was obtained by integration over 16 frames at a scan speed of 5 frames per second)

cules. In the absence of corrugations higher than a few $\AA$ it is possible to turn to the constant height mode by increasing the scanning speed and the time constant of the tip distance regulation. A reduction of the tip distance by increasing the average tunneling current or decreasing the bias voltage results in high resolution STM images. A high resolution STM image is shown in Fig. 2, where 16 frames were averaged at a scanning speed of 5 frames $/ \mathrm{s}$ with $I=1 \mathrm{nA}$ and $U=300 \mathrm{mV}$ tunneling current and voltage, respectively. The elongated form of individual PTCDA molecules, with an apparent double ring like structure at this resolution, is easily recognized. The unit cell of the periodic herringbone structure is marked by a rectangle in Fig. 2. The long axes of the two molecules in the unit cell, symmetry-related to one another by a (perpendicular) glide mirror plane, make an angle of $\sim 99^{\circ}$, on the average, in most parts of the figure $\left(\sim 49.5^{\circ}\right.$ to $b^{\prime}$, cf. Fig. 3), similar to that in the bulk $\alpha$-PTCDA crystal structure $\left(48.9^{\circ}\right.$ to $[\overline{2} 01]$ in the (102) plane, cf. [18]). In addition there are two regions (in the lower left and in the upper right corner) where a somewhat different mutual angle appears $\left(\sim 107^{\circ}\right.$ and $\left.\sim 90^{\circ}\right)$. The image displays a periodic intensity modulation in the horizontal direction. Every third vertical row of translationally equivalent molecules appears brighter. We attribute this modulation of the tunneling current to a superposition of influences from the underlying graphite surface lattice.

In a next step we increased the tunneling current further until the tip touched the organic film and thereby dragged the PTCDA molecules away; the underlying graphite lattice became visible and could be used for

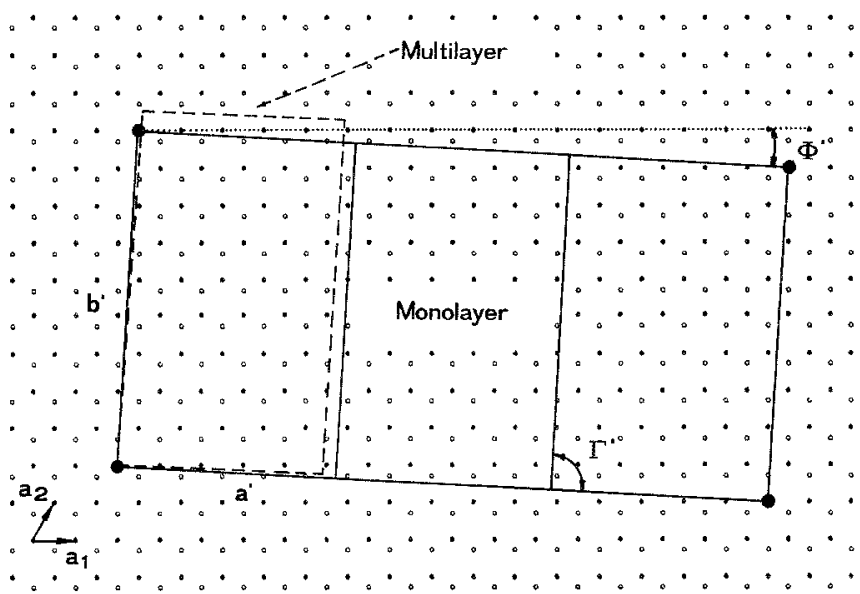

Fig. 3. Schematic presentation of the coincident PTCDA $1 \times 3$ superstructure on graphite (0001) and comparison of the twodimensional PTCDA unit cells in the monolayer and multilayer regime

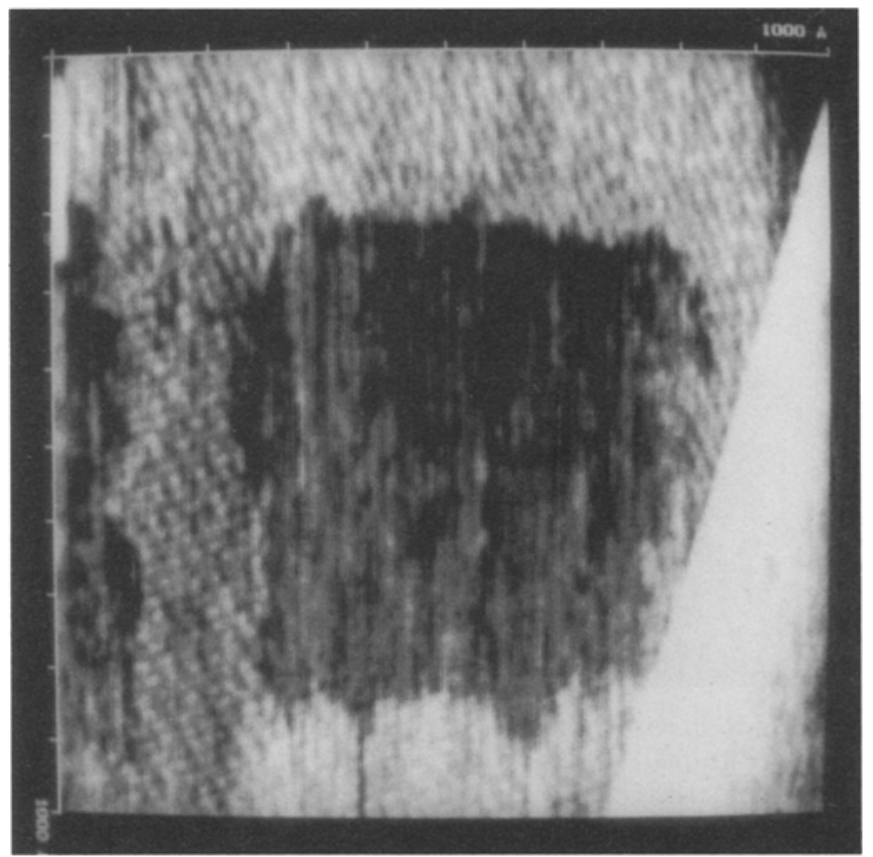

Fig. 4. A $1000 \AA \times 1000 \AA$ image of a PTCDA thin film on graphite with a $500 \AA \times 500 \AA$ window where the molecules were dragged away by the tunneling tip in a preceeding frame scanned at reduced tip distance

scale calibration. The lattice constants $a^{\prime}, b^{\prime}$, the angle $\Gamma^{\prime}=\Varangle\left(a^{\prime}, b^{\prime}\right)$, and the relative orientation $\Phi^{\prime}$ of the two-dimensional unit cell of the PTCDA overlayer (cf. Fig. 3) could thus directly be determined by comparison with the translational periods $\mathbf{a}_{1}$ and $\mathbf{a}_{2}$ of the graphite lattice. We obtained $\mathbf{a}^{\prime}=51 / 3 \mathbf{a}_{1}-1 / 3 \mathbf{a}_{2}, a^{\prime}$ $=12.7 \AA, \mathbf{b}^{\prime}=-4 \mathbf{a}_{1}+9 \mathbf{a}_{2}, b^{\prime}=19.2 \AA, \Gamma^{\prime}=89.5(1.0)^{\circ}$ and $\Phi^{\prime}=-3.2^{\circ}$, which can be abbreviated in the conventional representation as graphite (0001)-

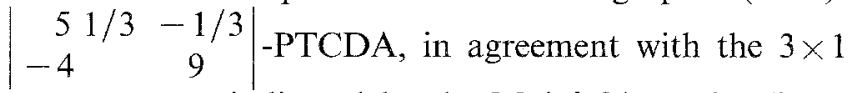
superstructure indicated by the Moire fringes in Fig. 2. The area of the unit cell, $F=\left|\mathbf{a}^{\prime} \times \mathbf{b}^{\prime}\right|$, amounts to $244 \AA^{2}$.

The image of Fig. 4, subsequently taken with the tunneling current decreased again, shows the window where 

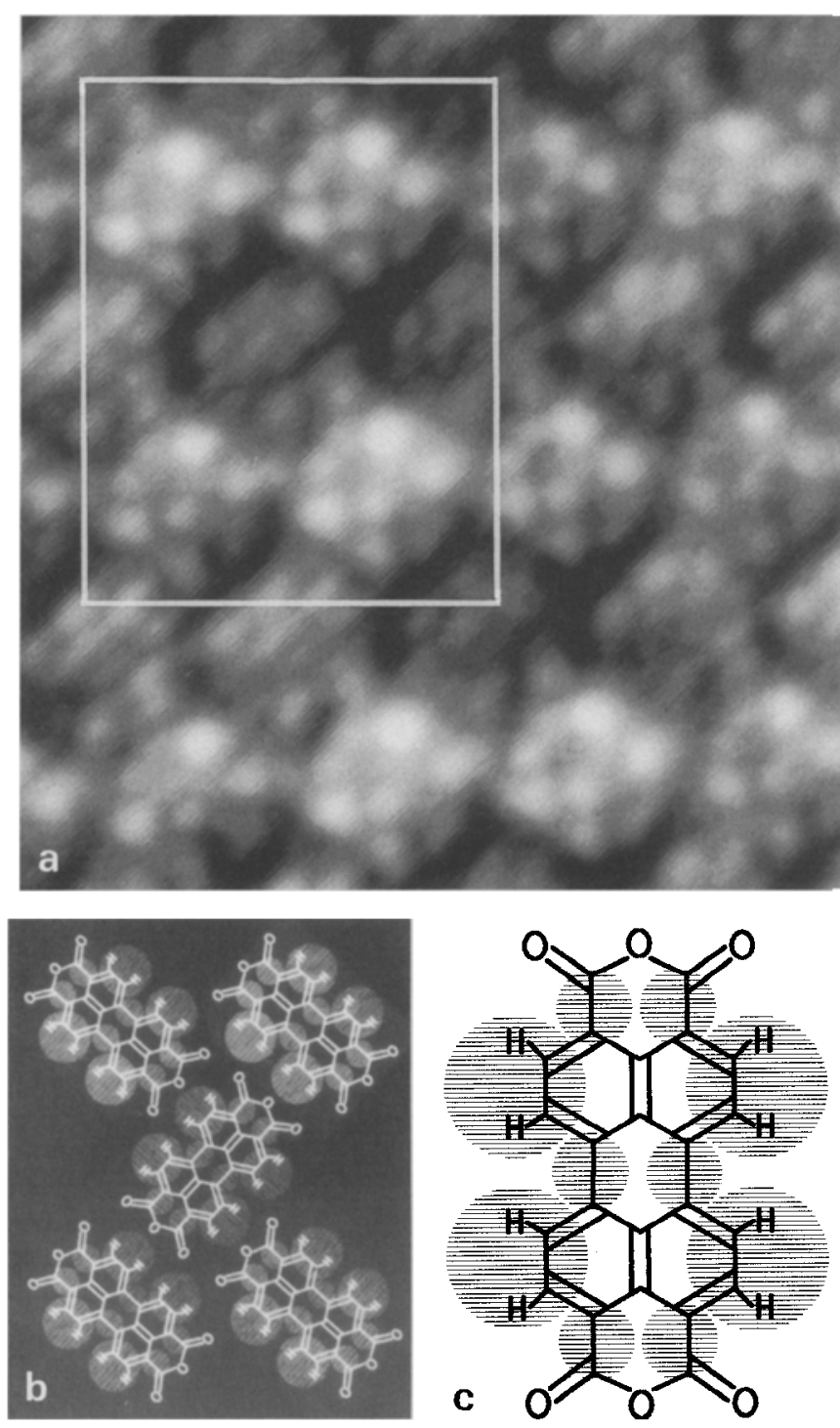

Fig. 5. a High resolution Video-STM image of a PTCDA monolayer on graphite (0001) with submolecular resolution, displaying intramolecular structures; each PTCDA molecule appears as a pattern of 10 bright spots, which are maxima of the tunneling current $(I=0.5 \mathrm{nA}, U=180 \mathrm{mV})$. b Correlation of the structure of the PTCDA molecules with maxima of the tunneling current, plotted for the marked area of Fig. 5a. c Enlarged view of the correlations of the molecular structure of PTCDA with the positions of the observed maxima of the tunneling current

clean graphite was uncovered in this way, while the initial overlayer still exists at the periphery.

While the dimensions and the orientation of the PTCDA unit cell could be determined with high precision in this way, the exact initial position of an individual molecule relative to the graphite atoms cannot be obtained because of a small drift and minor changes of the tip by touching the film between the scan of the complete organic film and the scan of the cleared substrate.

A tunneling current close to the critical value mentioned before leads to STM images with submolecular resolution (Fig. 5a). The double ring structures of Fig. 2, representing individual PTCDA molecules on the graph- ite surface, resolve into a pattern of ten bright spots ( $\max$ ima of the tunneling current), four larger and six smaller ones. This submolecular structure can be obtained in a wide range of bias voltages up to $0.3 \mathrm{~V}$ and is nearly independent of polarity. With the atomic coordinates, bond lengths and angles in the PTCDA molecule known from a full X-ray structure analysis [18] and the dimension of the unit cell taken from our calibrated STM images we were able to relate the bright spots to the molecular structure in the way plotted in Fig. $5 \mathrm{~b}$ and $5 \mathrm{c}$ (enlarged). It is remarkable that the six oxygen atoms are invisible in the STM pattern, that the centers of the four brighter spots lie between intramolecular pairs of hydrogen atoms and that the six smaller and weaker spots close to the long axis of the molecule are positioned between two carbon atoms each.

\subsection{LEED results}

Two slightly different types of LEED patterns as shown in Fig. $6 \mathrm{a}$ and $6 \mathrm{~b}$ were observed for monolayer and multilayer $(200 \AA)$ coverages of PTCDA deposited on graphite $(0001)$. The electron beam energy was $12.0 \mathrm{eV}$ in one and $13.0 \mathrm{eV}$ in the other pattern.

The absolute and, more precisely, the relative unit cell parameters of the two-dimensional surface lattice and the relative orientation of the unit cell with respect to the underlying graphite lattice are determined from the LEED diffraction patterns.

For the unit cell of the PTCDA monolayer structure we derive the relative size and orientation as shown in Fig. 7. Sketched is a graphite (0001)$\left|\begin{array}{rr}5.24(26) & -0.31(02) \\ -4.08(20) & 8.97(45)\end{array}\right|$-PTCDA unit cell with $\Gamma^{\prime}=$ $90.0(5)^{\circ}$ and $\Phi^{\prime}=-3.0(5)^{\circ}$ which represents one of six equivalent domains. These parameters can be obtained with an accuracy as indicated $( \pm 5 \%)$. From these relative cell dimensions the absolute ones are derived as $a^{\prime}$ $=12.5(6) \AA, b^{\prime}=19.1(9) \AA, F=239 \AA^{2}$, using the known graphite lattice parameters. The $a^{\prime}$ and $b^{\prime}$ values obtained are between those of the $\alpha$-form [18] and those of the $\beta$ form [19] of PTCDA, cf. Table 1. The distinct spot splitting is attributable to a certain deviation of the overlayer structure from an exact quasi-hexagonal symmetry.

From the observed extinctions of the $(0 k)$ and (h0)-reflections the existence of two orthogonal sets of glide symmetry planes parallel to the $a^{\prime}$ and $b^{\prime}$ axis is derived, which severely restrict the possible relative orientiations of the molecules in the unit cell, see e.g. [20].

For a thicker film $(200 \AA)$, PTCDA forms a graphite (0001)- $\left|\begin{array}{rr}5.00(25) & -0.25(01) \\ -4.39(22) & 9.49(47)\end{array}\right|$-PTCDA superstructure with lattice parameters $a^{\prime}=12.0(6) \AA, b^{\prime}=$ $20.2(1.0) \AA, \Gamma^{\prime}=90.0(5)^{\circ}, \Phi^{\prime}=-2.5(5)^{\circ}$, and $F=242 \AA^{2}$, which are close to the lattice parameters in the (102)plane of the $\alpha$-PTCDA crystal structure. This two-dimensional unit cell of the multilayer is compared in Fig. 3 with that of the monolayer structure.

The LEED and STM results are collected in Table 1 and compared with the parameters of the (102) plane of the $\alpha$-and of the $\beta$-modification. 

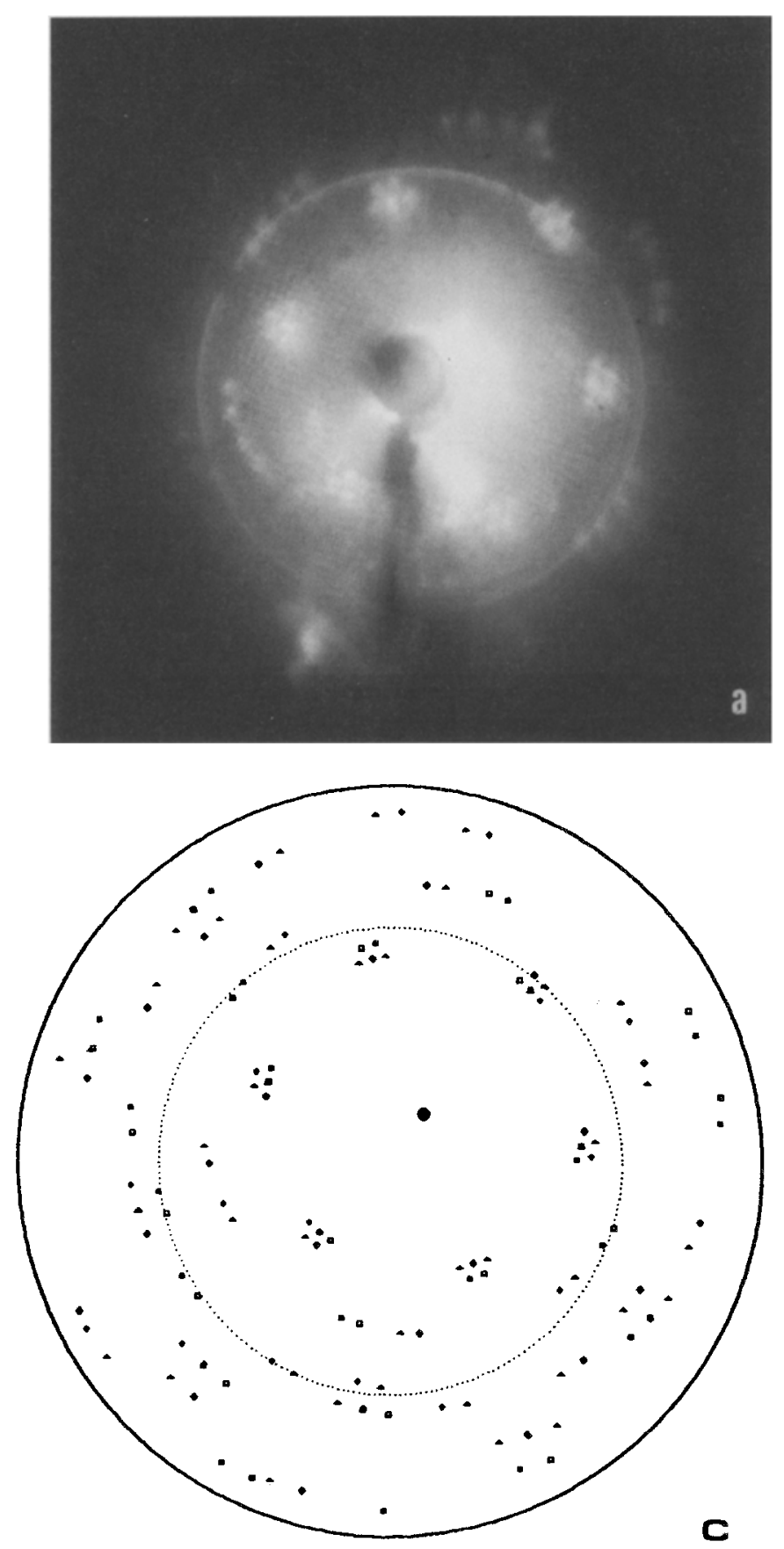

\subsection{Results of $X$-ray diffraction measurements}

$\mathrm{X}$-ray diffraction was detectable for a film thickness down to $100 \AA$. Scans taken in Bragg-Brentano geometry, i.e. measuring the separation of lattice planes parallel to the substrate, are displayed in Fig. 8 for two PTCDA films with a thickness of $100 \AA$ and $1000 \AA$, respectively. Apart from the very strong graphite reflection, only a single reflection attributable to $\alpha$-PTCDA could be detected, which appears exactly at the Bragg angle of the (102) reflection of the bulk crystal structure of $\alpha$-PTCDA, $2 \Theta_{(102)}^{(\alpha)}=27.7^{\circ}$. (In the $1000 \AA$ film an additional small contribution of the $\beta$-modification is indicated at $\left.2 \Theta_{(102)}^{(\beta)}=27.4^{\circ}\right)$. The rocking curve of the $(102) \alpha$-reflec-

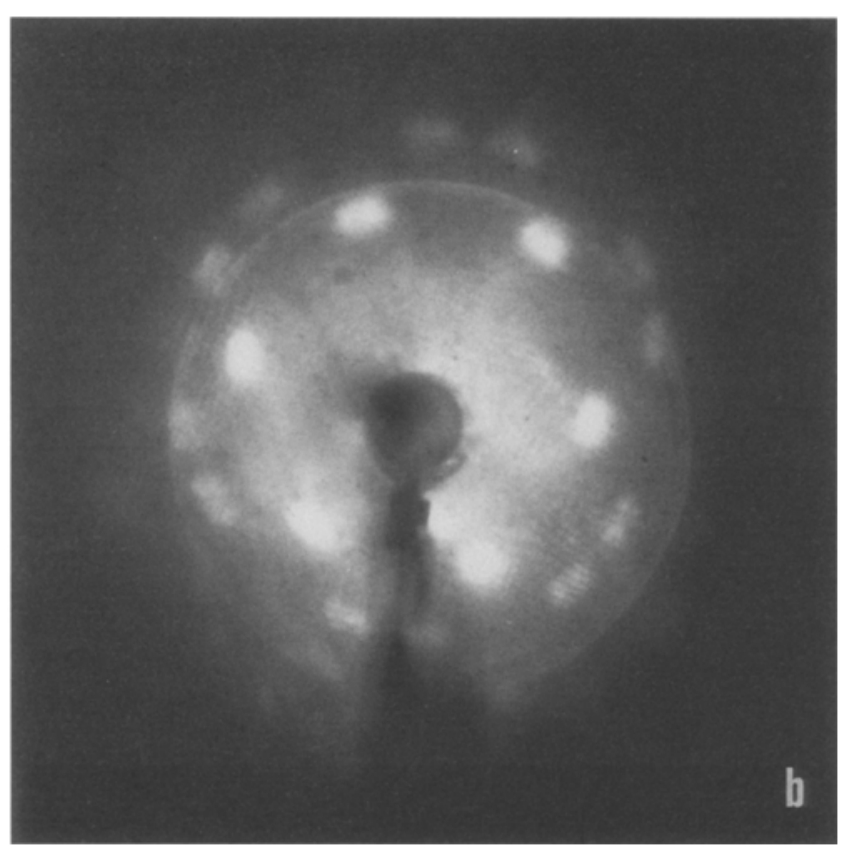

Fig. 6. LEED pattern at nearly normal incidence for (a) monolayer coverage and (b) multilayer coverage ( $200 \AA)$ of PTCDA on graphite $(0001)$, photographed through an image intensifier. The energy of the incident electron beam was $12.0 \mathrm{eV}$ in (a) and $13.0 \mathrm{eV}$ in (b). The ring around the center of the electron gun as well as the radial distortion of the pattern are artefacts produced by the image intensifier. (c) Geometrical calculation of the LEED-pattern expected for a monolayer of PTCDA on graphite (0001) with the structure proposed in Fig. 7, including all six symmetry-equivalent domain orientations. Due to glide symmetry elements, the $(h 0)$ and $(0 k)$ reflections are extinguished. The ring and the radial distortions have been taken into account in the calculation to allow a realistic comparison

tion has a remarkably narrow width of only $\pm 0.3^{\circ}$. It is therefore concluded that PTCDA grows very uniformly on graphite (0001) with the molecular planes parallel to the substrate surface even for thicker films. The $\alpha-(102)$ reflection of the $100 \AA$ film is broader than that of the thicker film; this can be attributed to the smaller number of reflecting planes which contribute to the interference pattern; the reflection also appears slightly shifted to smaller $2 \Theta$ angles, indicating a slightly increased interplanar distance. This can be taken as an indication of a somewhat different crystal structure in the first layers which is closer to the $\beta$-modification; a conclusion which is similar to that drawn from the LEED and STM results.

Films thicker than $100 \AA$ can be examined under a 
Table 1. Collection of the unit cell parameters of $\alpha$-and $\beta$-PTCDA, and comparison of the dimensions of their (102) unit meshes with those of the epitaxial thin films observed by LEED and STM. The unit cell parameters of the $\alpha$-modification are those obtained by a full $X$-ray crystal structure analysis [18]; the parameters of the $\beta$-modification were derived from X-ray powder data [19]. The hatched cross between the $\alpha$ and the $\beta$ colum indicates that, for reasons of a symmetry-consistent choice of the crystallographic axes of the two polymorphs, $b_{\alpha}^{\prime}$ is related to $a_{\beta}^{\prime}$, and $a_{\alpha}^{\prime}$ to $b_{\beta}^{\prime}$, cf. [19]

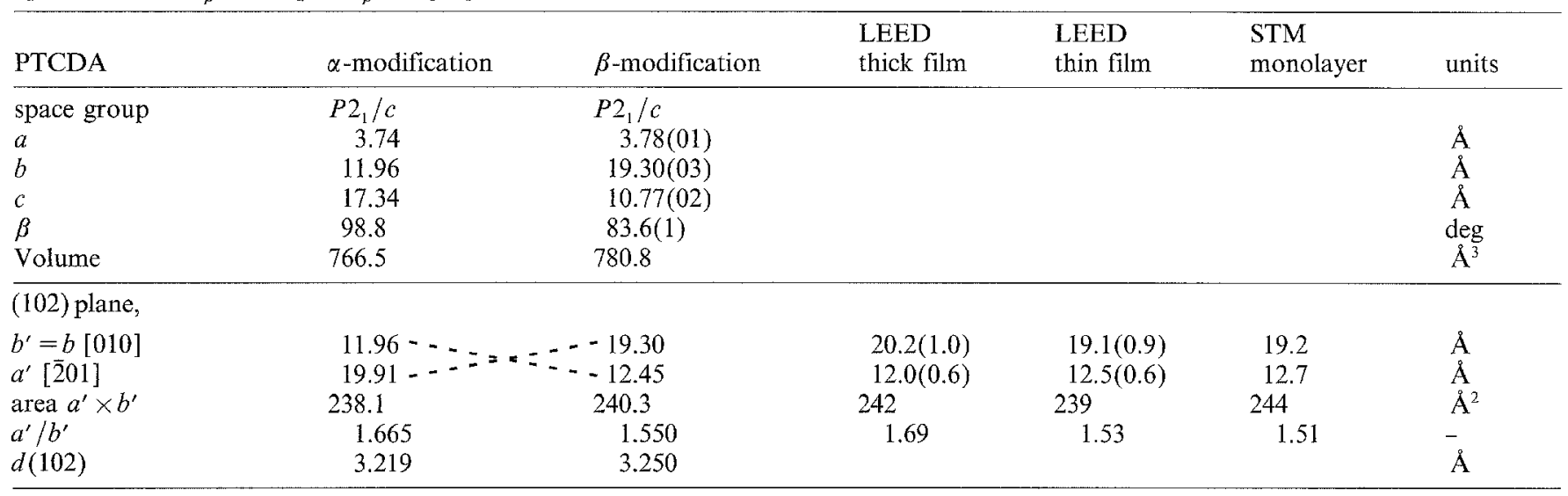

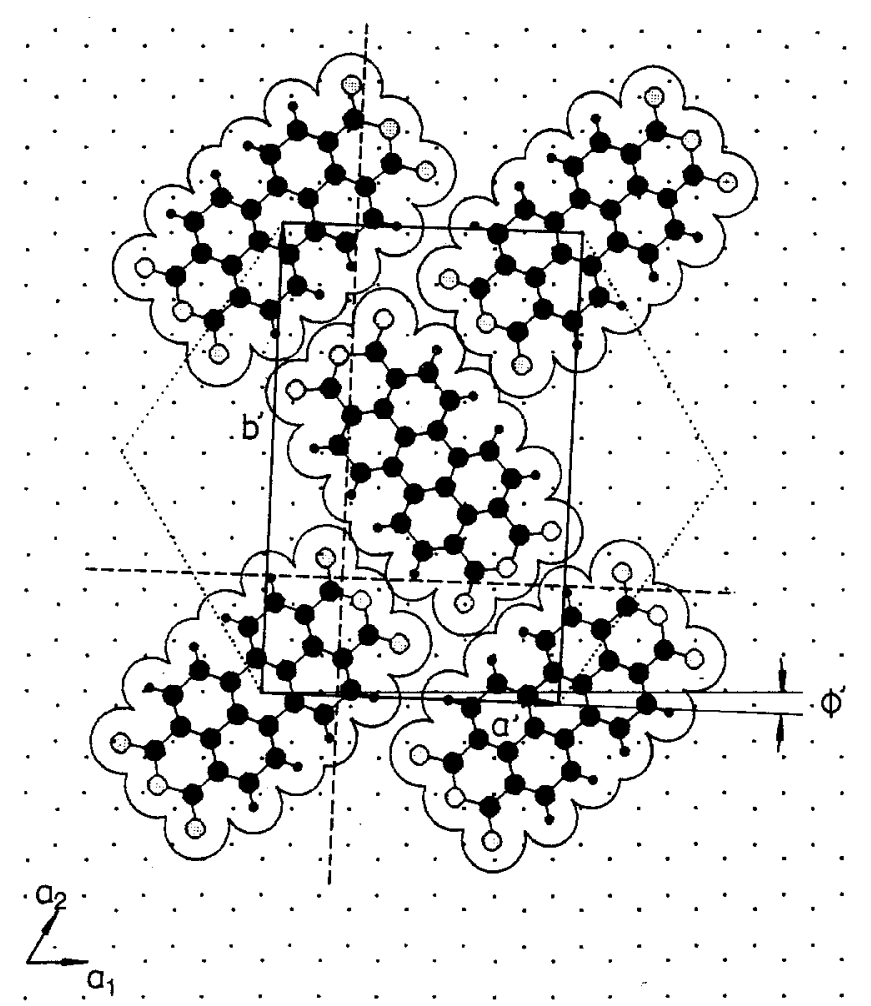

Fig. 7. Structural model of the ordering of a monolayer of PTCDA on graphite $(0001)$. Only one of the six equivalent domains is plotted. The glide symmetry elements of the space group $p 2 \mathrm{gg}$ are indicated as dashed lines. The dotted lines visualize the nearly quasihexagonal symmetry of the overlayer structure. Details about the molecular orientation with respect to the translational vectors of the overlayer lattice have been derived from symmetry considerations, see text

polarization microscope. A layer of very homogeneous thickness can be observed with no indication of island growth, not even at a deposition (substrate) temperature as high as $300^{\circ} \mathrm{C}$. This is in strong contrast to the behaviour of epitaxial PTCDA films on alkali halide crystal surfaces, where island growth prevails [19].

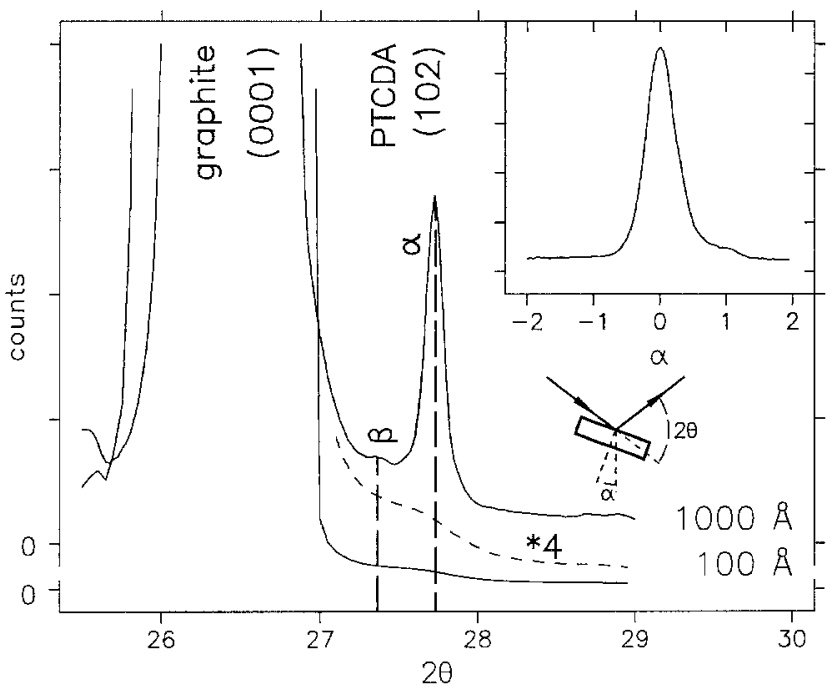

Fig. 8. X-ray diffraction pattern in Bragg-Brentano geometry of epitaxial PTCDA thin films of $100 \AA$ and $1000 \AA$ thickness on a graphite (0001) substrate. The inset shows a rocking curve whose narrow half width proofs the epitaxial order for two dimensions. Abscissa scales are in angular degrees

\section{Discussion}

In the subsequent discussion it will be shown that the results obtained by LEED and X-ray investigations in reciprocal space and by STM in direct space for PTCDA thin films prepared by molecular beam epitaxy on graphite (0001) complement one another in an ideal way to yield a very congruent structural picture. The unit cell dimensional and orientational parameters of the monolayer as obtained from the STM and from the LEED results are the same within the experimental accuracy. Due to the formation of Moire fringes in the STM images these cell parameters are taken as the more accurate ones.

There are several important conclusions which can be drawn from the experiments:

(1) The results described show that there exist well-defined preparation conditions which permit epitaxially 
oriented growth of PTCDA on graphite (0001) for coverages ranging from the monolayer to the multilayer region. (2) The detailed structure of the epilayer can be derived. We use the parameters of the two-dimensional surface lattice as obtained from the STM images and from the LEED patterns including the existence of the glide symmetry planes as obtained from the extinctions in the LEED patterns. The molecular size and shape is known from the X-ray crystal structure analysis [18]. We assume that the molecules lie flat on the surface (as inferred from the STM images, as well as from recent angular-dependent NEXAFS results [21] and, independently, with high precision from the Bragg-Brentano X-ray diffraction results, see Sect. 3.3). Further we assume that the molecules interact laterally by their polar side groups in a closest packing arrangement. Under these constraints there is only little room for structural variability. The area of the surface unit mesh found is close to that known for the bulk (102) plane, which amounts to $238 \AA^{2}$ for the $\alpha$ modification and $240 \AA^{2}$ for the $\beta$-modification. It can therefore be taken as confirmed that there are two PTCDA molecules in the two-dimensional surface unit cell, as already indicated by the high resolution STM images.

Close packing of two molecules in the unit cell in conjunction with the observed glide symmetry planes requires a zig-zag arrangement of the molecules. To find the optimal orientation we computed molecular plots. Starting from a mutual angle of $90^{\circ}$ between the long molecular axes we varied the angle, with the molecular centers fixed to the edges of the unit cell, until parts of the molecules came to overlap. Even with van der Waals radii slightly reduced in comparison with usual literature values $\left(r_{H}=1.1 \AA\right.$, instead of $1.2 \AA, r_{O}=1.3 \AA$ instead of $1.4 \AA)$ the exclusion of atomic overlap restricted the permissible variation of the relative orientation of the two symmetry-related PTCDA molecules to the narrow range of $90^{\circ} \pm 10^{\circ}$. A more rigorous derivation of these orientations as well as a determination of the absolute positions of the molecules on the surface relative to the individual substrate atoms would require LEED calculations on the basis of dynamical diffraction theory which are not accessible to us at present.

(3) From the STM results as well as from the LEED data, which mutually agree within the experimental error span, it is found that in the monolayer regime PTCDA forms a coincident $3 \times 1$ superstructure on the graphite (0001) surface with coincidence for every molecule in the $b^{\prime}$ direction and every third molecule in the $a^{\prime}$ direction with a translationally equivalent graphite site, which necessitates an angle $\Phi^{\prime}=-3.2^{\circ}$. A cell containing the overlayer unit cell can be chosen which has quasihexagonal symmetry when only the molecular positions are taken into account, cf. the dotted cell in Fig. 7; i.e. the superstructure unit cell has nearly the same symmetry as the substrate surface unit cell; the slight rotation by $\Phi^{\prime}=-3.2^{\circ}$ with respect to the latter leads to the formation of the observed multiple domains. A useful measure for the deviation from hexagonal symmetry is the ratio $b^{\prime} / a^{\prime}$ of the overlayer unit cell. For ideal hexagonal symmetry $b^{\prime} / a^{\prime}$ should be $\sqrt{3}=1.732$. In the monolayer structure $b^{\prime} / a^{\prime}=1.53(4)$ (yielding an angle $113.6^{\circ}$ instead of $120^{\circ}$ ), i.e. the deviation from ideal quasihexagonal symmetry is larger than in the case of the multilayer structure where $b^{\prime} / a^{\prime}=1.69(5)$. This slight symmetry-misfit for monolayer coverages is manifested in a distinct splitting of the LEED spots. For multilayer coverages the unit cell symmetry is closer to hexagonal. This is visible in a reduction of the splitting separations of the LEED spots, see Fig. $6 \mathrm{~b}$.

Comparing the ratio of the lattice constants for the $\alpha$ - and $\beta$-modification of the PTCDA crystal structure, $b^{\prime} / a^{\prime}=1.65$ and $b^{\prime} / a^{\prime}=1.55$, respectively, a pronounced flexibility in the molecular arrangement can be inferred, which leads to the build up of different polymorphs of PTCDA. In the case of PTCDA adsorbed on graphite (0001), where a distinct interaction with the substrate plays an important role, this flexibility seems to be of particular importance for the formation of a well ordered monolayer, which then acts as the basis for further growth to a well ordered homogeneous thicker film: a comparison of the (102)-plane of the bulk PTCDA crystal structure and the (0001) surface of graphite shows that no arrangement of the ideal $\alpha$ - or $\beta$-PTCDA (102)-plane on graphite (0001) can be found where the PTCDA molecules occupy surface lattice sites fully commensurate with the graphite surface lattice, i.e. lattice constant fit with the bulk PTCDA structures cannot be obtained. Rather, commensurability (more precisely "coincidence") which plays an important role for the order and stability of the monolayer structure, is achieved by a structural adaption of the overlayer. From lower to higher coverages the influence of the substrate lattice decreases and the $\alpha$-modification is gradually approached, cf. Fig. 6 b. Due to the layered PTCDA crystal structure, well ordered, homogeneous films in the $\alpha$-modification can finally form, instead of the frequently encountered island growth. This is confirmed by optical microscopy.

(4) For a more detailed interpretation of the high resolution STM results a discussion of the mechanism of STM contrast formation is necessary.

In the theoretical treatment of STM imaging by Tersoff and Hamann [22] the local distribution of the tunneling current density is determined by the local density of surface states at the Fermi level. For the STM image of a molecule it is therefore expected from this theory that the tunneling current distribution corresponds to maxima of the electron densities of the molecule. Alternatively it has been proposed by Spong et al. that a modulation of the local work function of the substrate by insulating polarizable molecular adsorbates can explain the observed molecular images [23]. This mechanism would also require high electron densities at the maxima of the tunneling current. Instead we find six weaker and four larger bright spots, see Fig. 5, which must be attributed to positions with lower electron density. It is remarkable that the six weaker spots have positions where the $\mathrm{C}-\mathrm{C}$ bond lengths of the PTCDA molecule amout to $1.46 \AA$, which is significantly longer than the average of $1.38 \AA,[18]$. In the framework of the Hückel Molecular Orbital method [24] applied to conjugated $\pi$-electron systems, an increased bond length corresponds to a higher potential energy of the electrons between the two C-atoms and a reduced electron density. Thus the maxima of the 
tunneling current at the six weaker spots are apparently caused by an elevated potential which reduces the effective barrier height for tunneling to the tip.

At the positions of the four bright maxima at the hydrogen ligands the electron density is reduced as a consequence of the more electropositive nature of hydrogen. Therefore electrons in this region also see an elevated potential and a correspondingly reduced barrier height. Besides the possibility that the barrier height is reduced in these areas, the hydrogen ligands could be bent up from the molecular plane, or exhibit strong zero point or thermal motion in vertical direction; this would reduce the effective distance to the tunneling tip and thereby increase the tunneling current.

The fact that the six oxygen atoms are not visible in the STM images might be related to their strong electronegativity (tendency to attract electrons). It is worth mentioning that CO-molecules, coadsorbed with benzene molecules on Rh (111) also did not show up in STM images [4]. The electronic states of the adsorbate and local changes of the work function were considered as possible causes [4]. Alternatively, for the large PTCDA molecule, a bending down of the ligands by specific oxygen interactions with the substrate $\mathrm{C}$-atoms might be responsible. This would conform with the observed stronger binding of the PTCDA molecule to a graphite substrate as compared with an unsubstituted aromatic hydrocarbon, such as e.g. coronene, for which we could not detect an ordered molecular arrangement by STM at room temperature, a fact which we attribute to a too high molecular mobility on the graphite substrate surface.

In any case, our results indicate that the interpretation of the molecular STM images of PTCDA is not possible in terms of the local densities of states at the Fermi level, as predicted by the theory of Tersoff and Hamann, which is based on the many particle tunneling description developed by Bardeen [24]. Instead, our tentative interpretation of the observed STM image contrast of PTCDA appears to be related to the independent particle model of tunneling formulated by Harrison [25], which does not contain the electronic density of states.

The solution of these questions requires further experimental data and theoretical considerations.

\section{Conclusions}

PTCDA on graphite (0001) is, to the best of our knowledge, the first organic epitaxial thin film system for which the detailed crystallographic structure has been determined from the mono- to the multilayer regime by several independent methods, LEED, X-ray diffraction and STM. These different methods yielded congruent results. Our investigations show that there exist well-defined preparation conditions which permit epitaxial growth of films of thicknesses ranging from the monolayer regime to several hundred $\mathrm{nm}$. The LEED and STM values for the dimensions of the unit cell of monolayers agree well and are close to the lattice parameters in the (102)-plane of the bulk crystal structure of the newly found $\beta$-modification. The overlayer structure is coincident with the graphite surface with positional coincidence for every third molecule. With the used video-STM we were able to obtain submolecular resolution. The observed molecular image cannot be explained by density of states arguments; an independent particle model of tunneling seems to be more appropriate.

The authors would like to thank Dr. K. Laßmann for helpful discussions. Highly purified PTCDA was kindly placed at our disposal by Prof. Dr. H. Langhals, Institut für Organische Chemie, Universität München, and further purified by temperature gradient sublimation by Miss S. Weinmann in our Crystal Laboratory. We are greatly indebted to Prof. S.R. Forrest for making available to us unpublished tables of the PTCDA X-ray crystal structure analysis, containing atomic positions, thermal parameters, bond lengths and closest contacts. The natural graphite crystals were a kind gift by Dr. A. Moore from Union Carbide Company. Financial support by the Deutsche Forschungsgemeinschaft via Sonderforschungsbereich 329 is gratefully acknowledged.

\section{References}

1. Zimmermann, U., Schnitzler, G., Karl, N., Umbach, E., Dudde: Thin Solid Films 175, 85 (1989)

2. Zimmermann, U., Schnitzler, G., Schneider, M., Kaiser, M., Herde, R., Wüstenhagen, V., Taborski, J., Karl, N., Dudde, R., Koch, E.E., Umbach, E.: Vacuum 42, 1795 (1990)

3. Ohtani, H., Hove, M.A. van, Somorjai, G.A.: J. Vac. Sci. Technol. A6, 633 (1988)

4. Ohtani, H., Wilson, R.J., Chiang, S., Mate, C.M.: Phys. Rev. Lett. 60, 2398 (1988)

5. Dahlgren, D., Hemminger, J.C.: Surf. Sci. 114, 459 (1982)

6. Gland, J.L., Somorjai, G.A.: Surf. Sci. 38, 157 (1973)

7. Hallmark, V.M., Chiang, S., Brown, J.K., Wöll, Ch.: Phys. Rev. Lett. 66, 48 (1991)

8. Yannoulis, P.: Thesis, Universität Hamburg 1988

9. Buchholz, J.C., Somorjai, G.A.: Chem. Phys. 66, 573 (1977)

10. Gimzewski, J.K., Stoll, E., Schlittler, R.R.: Surf. Sci. 181, 267 (1987)

11. Firment, L.E., Somorjai, G.A.: J. Chem. Phys. 66, 2901 (1977)

12. Somorjai, G.A.: Chemistry in two dimensions: surfaces. Ithaca: Cornell 1981

13. Zimmermann, U., Karl, N.: Surf. Sci. (in print)

14. Rabe, J.P., Buchholz, S.: Phys. Rev. Lett. 66, 2096 (1991); idem, Makromol. Chemie - Macromol. Symposia, BPS'91 (in press)

15. Forrest, S.R., Kaplan, M.L., Schmidt, P.H.: J. Appl. Phys. 55, $1492(1984)$

16. Forrest, S.R., Leu, L.Y., So, F.F., Yoon, W.Y.: J. Appl. Phys. 66, 5908 (1989)

17. So, F.F., Forrest, S.R.: IEEE Trans. Electron. Dev. 36, 66 (1988)

18. Lovinger, A.J., Forrest, S.R., Kaplan, M.L., Schmidt, P.H., Venkatesan, T.: J. Appl. Phys. 55, 476 (1984); for PTCDA crystal structure data see also: idem, Bull. Am. Phys. Soc. 28, 363 (1983); full set of crystal structure data: Kaplan, M.L., Day, C.S., Lovinger, A.J., Schmidt, P.H., Forrest, S.R.: (Private communication)

19. Möbus, M., Karl, N., Kobayashi, T.: J. Cryst. Growth (in print)

20. Vainshtein, B.K.: Structure analysis by electron diffraction. Oxford, New York: Pergamon Press 1964

21. Möbus,. M., Schmelzer, M., Taborski, J., Zimmermann, U., Karl, N., Umbach, E.: Unpublished results

22. Tersoff, J., Hamann, D.R.: Phys. Rev. B31, 805 (1985)

23. Spong, J.K., Mizes, H.A., LaComb, L.J. Jr., Dovek, M.M., Frommer, J.E., Foster, J.S.: Nature 338, 137 (1989)

24. Försterling, H.D., Kuhn, H.: Moleküle und Molekülanhäufungen. Berlin, Heidelberg, New York: Springer 1983

25. Bardeen, J.: Phys. Rev. Lett. 6, 57 (1961)

26. Harrison, W.A.: Phys. Rev. 123, 85 (1961)

27. Karl, N., Sato, N.: Mol. Cryst. Liq. Cryst. (submitted for publication) 